\title{
HEISENBERG ACTION IN SKEIN THEORY AND EXTERNAL EDGE CONDITION
}

\author{
Hajime FUJITA
}

(Received 6 April 2009)

\begin{abstract}
In this paper we give an explicit description of the representation matrix of a Heisenberg type action previously constructed by Blanchet et al. Our construction is based on the combinatorial part of Yoshida's construction. We give the matrix in terms of a ribbon graph and its admissible colorings. We show that the components of the representation matrix satisfy the external edge condition, which is a natural combinatorial/geometric condition for maps from the first homology of the graph. We give the explicit formula of the trace of the action in the case of surfaces with colored structure using the external edge condition, the Verlinde formula and elementary counting arguments. Our formula is a generalization of the results for a surface without colored structure, which are already known.
\end{abstract}

\section{Introduction}

In [5], Blanchet et al constructed a family of topological quantum field theories (TQFT) parameterized by non-negative integers $p$ as a functor from a two-dimensional cobordism category $\dagger$ to the category of modules. They constructed a TQFT-module $V_{p}(C)=$ $V_{p}\left(C, l, j^{\prime}\right)$ for each surface with colored structure $\left(C, l, j^{\prime}\right)$. A surface with colored structure is a triple $\left(C, l, j^{\prime}\right)$ consisting of a closed oriented surface $C$, a banded link $l=l_{1} \cup \cdots \cup l_{n}$ in $C$ (i.e. embedded disjoint intervals) and a set of fixed colorings $j^{\prime}=$ $\left(j_{1}^{\prime}, \ldots, j_{n}^{\prime}\right)$ of components $l_{1}, \ldots, l_{n}$. Here a coloring is an integer. On the other hand, for each admissible coloring of a ribbon graph (Definition 2.1), one can associate an element of the TQFT-module. They also showed that such elements form a free basis of their TQFTmodule. Our interest in this paper is the Heisenberg type action on the TQFT-module for $p=2 k+4$. They defined involutions on the TQFT-module $V_{2 k+4}(C)$ associated with simple closed curves on the surface, and these involutions form a natural action of a Heisenberg type group defined as a central extension of $H_{1}(C-l ; \mathbb{Z} / 2)$. Such a Heisenberg action is a starting point of a refinement of their TQFT, which is called spin-refined TQFT $[3,4]$.

The purpose of this paper is to have an explicit representation matrix of the Heisenberg action on the TQFT-module in terms of a given ribbon graph and its $k$-admissible colorings based on the combinatorial part of Yoshida's construction [8], and we carry out the computation of the trace of the action for a surface with colored structure using such an

2000 Mathematics Subject Classification: Primary 57R56; Secondary 57M25, 58D19, 05 C25.

Keywords: topological quantum field theory; TQFT; skein theory; Heisenberg action.

$\dagger$ The domain category considered in [5] is really that of surfaces with $p_{1}$-structure. In this paper we do not need any $p_{1}$-structure and we omit it for simplicity.

(C) 2010 Faculty of Mathematics, Kyushu University 
explicit description. The main ingredient for the computation of the trace is the external edge condition, which is a quite natural geometric/combinatorial condition for a map from the homology of the graph to the coefficient ring of the TQFT-module. The explicit description of the representation matrix tells us that the map appearing in the matrix satisfies the external edge condition. As a corollary of the computation of the trace we have the dimension formula for the brick decomposition of the TQFT-module for a surface with colored structure. The formula for a surface without a banded link is obtained in [2] and [5] using algebro-geometric and skein theoretical technique, respectively. Our explicit computation works uniformly for a surface with a non-empty link. The computation of the trace also tells us that the external edge condition is a characterization of the Heisenberg action on the TQFT-module (Remark 6.1).

We should note that Masbaum gave the computation of the representation matrix a long time ago but he never published it. Such a computation is a special case of the algorithm called TQFT that was implemented by Masbaum and A'Campo in the PARI program to compute representation matrices of the mapping class group on the TQFT-module. It is possible to compute representation matrices of the mapping class group using similar methods. See [1] for the program TQFT and its applications.

This paper is organized as follows. In Section 2 we recall some results obtained in [7], which we use in Section 3 to compute the representation matrix explicitly. In Section 3 we first recall the definition of the involutions on the TQFT-modules. We give explicit descriptions of involutions on the TQFT-module associated with a meridian cycle and a longitude cycle. In Section 3.1 we introduce the external edge condition (Definition 3.5). By making use of the explicit description one can check that the map appearing in the matrix satisfies the external edge condition (Proposition 3.4). In Section 4 we define four lattices associated with the graph and realize the first homology group of the surface in terms of these lattices (Proposition 4.2). These lattices are introduced in [8] for a closed surface with a pants decomposition. In Section 5 we first recall the definition of the Heisenberg type group in [5], and the explicit description obtained in Section 3 enables us to describe the action of the Heisenberg type group explicitly in terms of the graph and its $k$-admissible colorings. In Section 6 we compute the trace of the involution for a surface with colored structure (Theorem 6.1). To show the formula we use the external edge condition, the Verlinde formula and elementary counting arguments. In Section 7 we demonstrate the computation of the dimension formulas for a brick decomposition of the TQFT-module for a surface with colored structure.

Convention. In the figures, we use the convention that a line represents a ribbon parallel to the plane and each trivalent vertex is ordered in the counterclockwise direction (see Figure 1).

\section{Some results in the Temperley-Lieb algebra}

In this section we recall some results obtained in [7], which are used in the next section to obtain the explicit description of involutions on the TQFT-module. Fix a non-negative integer $k$. We first recall the definition of $k$-admissible colorings of a graph.

Definition 2.1. Let $\Gamma$ be a trivalent graph. A map $j$ from the set of edges of $\Gamma$ to the set of integers $\{0,1, \ldots, k\}$ is called a $k$-admissible coloring of $\Gamma$ if the following three conditions 


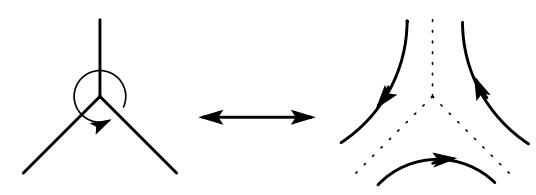

FIGURE 1. Convention of orientation.

hold at each vertex with edges $f_{1}, f_{2}$ and $f_{3}$ :

$$
\begin{aligned}
j\left(f_{1}\right)+j\left(f_{2}\right)+j\left(f_{3}\right) & \in 2 \mathbb{Z} ; \\
\left|j\left(f_{1}\right)-j\left(f_{2}\right)\right| \leq j\left(f_{3}\right) & \leq j\left(f_{1}\right)+j\left(f_{2}\right) ; \\
j\left(f_{1}\right)+j\left(f_{2}\right)+j\left(f_{3}\right) & \leq 2 k .
\end{aligned}
$$

For a vertex with two edges $f_{1}$ and $f_{2}=f_{3}$, we interpret these conditions as a corresponding condition with $j\left(f_{2}\right)=j\left(f_{3}\right)$. These three conditions are called the quantum ClebschGordan condition of level $k$.

For a unitrivalent graph $\Gamma$ with $n$ univalent vertices, we fix an $n$-tuple of colorings $j^{\prime}=\left(j_{1}^{\prime}, \ldots, j_{n}^{\prime}\right)$ (boundary coloring). A coloring $j$ of $\Gamma$ is $k$-admissible if it satisfies the quantum Clebsch-Gordan condition of level $k$ at each trivalent vertex and it is compatible with $j^{\prime}$, i.e. $j\left(f_{l}\right)=j_{l}^{\prime}$ for all $f_{l}$ with a univalent vertex.

The Jones-Kauffman skein module of a compact three-dimensional manifold $M$ over a commutative ring $R$ is defined as the $R$-module generated by the set of isotopy classes of banded links in $M$ meeting $\partial M$ transversally in the banded link in $\partial M$ quotiented by the Kauffman relations. A colored ribbon graph with a $k$-admissible coloring in a compact three-dimensional manifold gives rise to an element in the Jones-Kauffman skein module by expanding the graph at each vertex and inserting the Jones-Wenzl idempotent at each edge. See [5] for details. The coefficient ring of the TQFT-module in [5] is defined as a kind of cyclotomic ring of degree $2(2 k+4)$. It contains an indeterminate $A$, which should be a $2(2 k+4)$ th root of unity. Recall the following notation:

(i) $[n]:=\frac{A^{2 n}-A^{-2 n}}{A^{2}-A^{-2}}$;

(ii) $\quad[n] !:=[1][2] \cdots[n], \quad([0] !:=1)$;

(iii) $\langle n\rangle:=(-1)^{n}[n+1]$.

It is known that $[n]$ is invertible for $n=1,2, \ldots, k+1$ and $[k+2]=0$.

Now we recall the results in [7], which we will use in the next section. Those are equalities in the Temperley-Lieb algebra. The Temperley-Lieb algebra is the JonesKauffman skein module of the box $D^{2} \times[0,1]$ with the standard link in $D^{2} \times\{0\}$ and $D^{2} \times\{1\}$. The product is given by the standard product of tangles.

TheOrem 1. (Fusion coefficient) Put

$$
\langle a, b, c\rangle=(-1)^{i+j+k} \frac{[i+j+k+1] ![i] ![j] ![k] !}{[i+j] ![j+k] ![k+i] !},
$$


where $i, j$ and $k$ (internal colors) are defined by

$$
\begin{aligned}
& i=\frac{1}{2}(-a+b+c), \\
& j=\frac{1}{2}(a-b+c), \\
& k=\frac{1}{2}(a+b-c) .
\end{aligned}
$$

Then one has the following two equalities in the Temperley-Lieb algebra.

$$
\begin{aligned}
& \left.\right|_{a b b}=\sum_{c} \frac{\langle c\rangle}{\langle a, b, c\rangle} \underbrace{c}_{b} \\
& a \int_{c^{\prime}}^{c} b=\left.\delta_{c, c^{\prime}} \frac{\langle a, b, c\rangle}{\langle c\rangle}\right|^{c}
\end{aligned}
$$

TheOrem 2. (Tetrahedron coefficient) Put

$$
\left\langle\begin{array}{lll}
a & b & c \\
d & e & f
\end{array}\right\rangle=\frac{\prod_{i=1}^{3} \prod_{j=1}^{4}\left[b_{i}-a_{j}\right] !}{[a] ![b] ![c] ![d] ![e] ![f] !}\left(\begin{array}{cccc}
a_{1} & a_{2} & a_{3} & a_{4} \\
b_{1} & b_{2} & b_{3} &
\end{array}\right),
$$

where

$$
\left(\begin{array}{llll}
a_{1} & a_{2} & a_{3} & a_{4} \\
b_{1} & b_{2} & b_{3} &
\end{array}\right)=\sum_{\max \left(a_{j}\right) \leq \zeta \leq \min \left(b_{i}\right)} \frac{(-1)^{\zeta}[\zeta+1] !}{\prod_{i=1}^{3}\left[b_{i}-\zeta\right] ! \prod_{j=1}^{4}\left[\zeta-a_{j}\right] !}
$$

for

$$
\begin{aligned}
& a_{1}=\frac{1}{2}(a+b+c), \quad b_{1}=\frac{1}{2}(b+c+e+f), \\
& a_{2}=\frac{1}{2}(b+d+f), \quad b_{2}=\frac{1}{2}(a+b+d+e), \\
& a_{3}=\frac{1}{2}(c+d+e), \quad b_{3}=\frac{1}{2}(a+c+d+f), \\
& a_{4}=\frac{1}{2}(a+e+f) .
\end{aligned}
$$

Then one has the following equality in the Temperley-Lieb algebra.

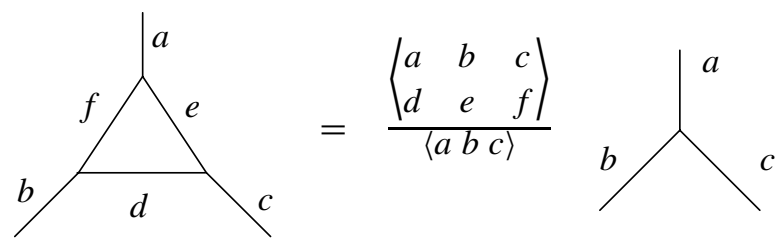




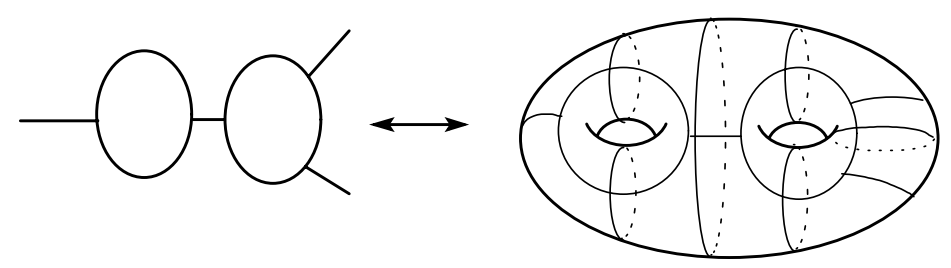

FIGURE 2. Ribbon graph and surface with a pants decomposition.

Theorem 3. (Half twist coefficient) Put

$$
\delta(c ; a, b)=(-1)^{k} A^{i j-k(i+j+k+2)},
$$

where $i, j$ and $k$ are as in Theorem 1. Then one has the following equality in the TemperleyLieb algebra.

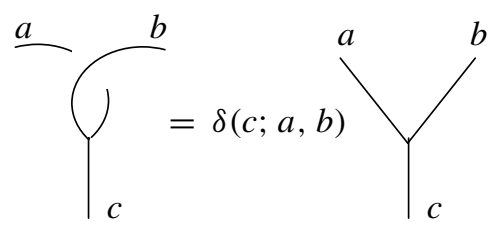

\section{Computation of representation matrices of the involutions}

In this section we give graphical computations for involutions on the TQFT-module and obtain the explicit description of them. The involution in [5] is defined as follows. For each simple closed curve $c$ on an oriented surface $C$, set $Z(c)_{0}:=\left(C \times[0,1], c_{1 / 2}(k)\right)$, where $c_{1 / 2}(k)$ is a knot $c \times\{1 / 2\}$ in $C \times[0,1]$ colored by $k$. Then $Z(c)_{0}$ is a cobordism from $C$ to itself and it induces an endomorphism on the TQFT-module $V_{2 k+4}(C)$. The involution in [5] is defined by $Z(c):=(-1)^{k} Z(c)_{0}$.

Now we fix a ribbon unitrivalent graph $\Gamma$. Let $B_{\Gamma}$ be the product of the interval $[0,1]$ and the ribbon associated with $\Gamma$, which is homeomorphic to a handle body. Let $C_{\Gamma}$ be the boundary of $B_{\Gamma}$. Note that $C_{\Gamma}$ is a closed oriented surface equipped with the pants decomposition which is dual to the given graph $\Gamma$, and $C_{\Gamma}$ may contain a banded link corresponding to univalent vertices of $\Gamma$ (see Figure 2). We also have a copy $\Gamma_{0}=\{0\} \times \Gamma \subset$ $C_{\Gamma}$ of $\Gamma$.

Fix a boundary coloring $j^{\prime}$ of $\Gamma$. For each $k$-admissible coloring $j$ of $\Gamma$, a triple $\left(B_{\Gamma}, \Gamma, j\right)$ defines an element of the TQFT-module $V_{2 k+4}\left(C_{\Gamma}\right)=V_{2 k+4}\left(C_{\Gamma}, j^{\prime}\right)$. In [5] the authors showed that the elements $\left\{\left(B_{\Gamma}, \Gamma, j\right) \mid j: k\right.$-admissible $\}$ form a free basis of $V_{2 k+4}\left(C_{\Gamma}\right)$. In this setting the involution explained above is described as

$$
Z(c):\left(B_{\Gamma}, \Gamma, j\right) \mapsto(-1)^{k}\left(B_{\Gamma} \cup\left(C_{\Gamma} \times[0,1]\right), \Gamma \sqcup c, j \sqcup k\right),
$$

where $c$ is a simple closed curve on $C_{\Gamma}$ and $j$ is a $k$-admissible coloring of $\Gamma$. In this section we have an explicit description of this involution for a meridian cycle and a longitude cycle. Here a meridian cycle means an element in the kernel of the natural map $H_{1}\left(C_{\Gamma}\right) \rightarrow H_{1}\left(B_{\Gamma}\right)$ 


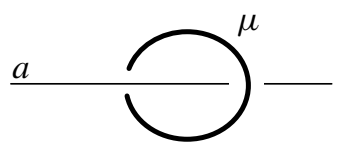

FIgURE 3. Meridian $\mu$.

and a longitude cycle means an element in $H_{1}\left(\Gamma_{0}\right)$. Before starting the computation we note the following equalities:

(i) $[k+2-\alpha]=[\alpha]$;

(ii) $\quad[k-\alpha] ![\alpha+1] !=[k+1] !$

(iii) $\frac{\langle k-a\rangle}{\langle a k k-a\rangle}=(-1)^{a}[a+1]=\langle a\rangle=(-1)^{k}\langle k-a\rangle$;

(iv) $\delta(k-a ; k, a)=(-1)^{a} A^{-a(k+2)}$;

(v) $\frac{\left(\begin{array}{ccc}k-A & k-B & C \\ B & A & k\end{array}\right)}{\langle k-A k-B C\rangle}=(-1)^{(A+B-C) / 2} \frac{[k-A] ![k-B] ![(A+B+C) / 2+1] !}{[k+1] ![k+1-(A+B-C) / 2] !}$.

All these formulas are shown by the direct computations, and the left-hand side of last three equalities appear in Theorems 1, 2 and 3 for a graph with an edge colored by $k$. We also note that a triple $(k, a, c)$ is $k$-admissible if and only if $c=k-a$.

Hereafter we denote an element $\left(B_{\Gamma}, \Gamma, j\right)$ by $|j\rangle$ for simplicity.

Meridian. Take a meridian circle $\mu$ or a circle around a component of the banded link. Fix a $k$-admissible coloring $j$ of $\Gamma$. Assume that $\mu$ has an edge colored by $a$ as its core. See Figure 3. Here the thick line represents the cycle colored by $k$, and we use such a notation throughout this paper. By the graphical computation below one has that

$$
Z(\mu):|j\rangle \mapsto(-1)^{a}|j\rangle .
$$

See also Remark 7.6 in [5]. In fact one can compute as follows.

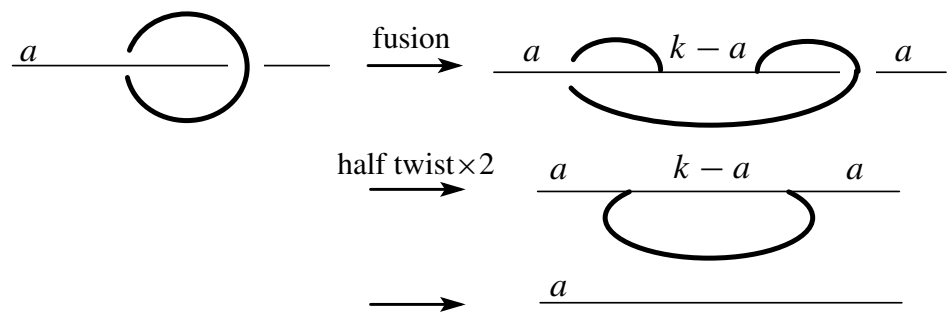

The coefficient associated with these diagrams is

$$
\frac{\langle k-a\rangle}{\langle a k k-a\rangle} \delta(k-a ; k, a)^{2} \frac{\langle a k k-a\rangle}{\langle a\rangle}=A^{-2 a(k+2)} \frac{\langle k-a\rangle}{\langle a\rangle}=(-1)^{k+a} .
$$



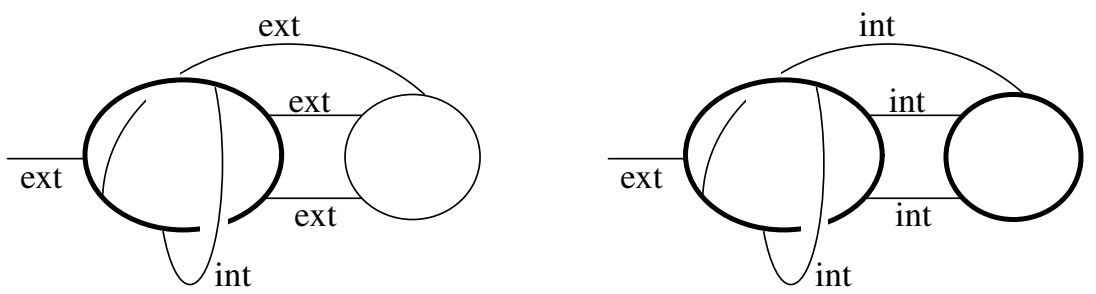

FIGURE 4. External edges and internal edges for $\lambda$ depicted by thick lines.

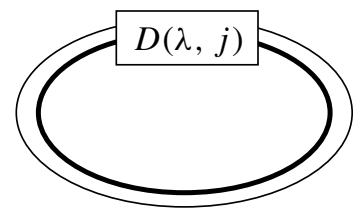

FIGURE 5. Diagram around $\lambda$.

Here we have used the equality

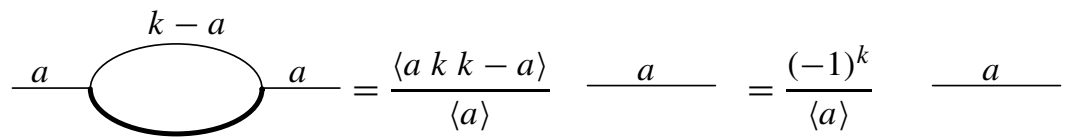

Longitude. Take a cycle $\lambda$ in $\Gamma_{0} \subset C_{\Gamma}$ and a $k$-admissible coloring $j$ of $\Gamma$. To compute the explicit description of the involution $Z(\lambda)$ on $|j\rangle$ it is enough to consider $\lambda$-external (respectively internal) edges.

Definition 3.1. ( $\lambda$-external edge and $\lambda$-internal edge) For a cycle $\lambda$ in $\Gamma$, an edge $f \in \Gamma$ is said to be a $\lambda$-external edge if the cycle $\lambda$ on $\Gamma$ does not pass through $f$ and one vertex of $f$ lies on $\lambda$ and the other does not. If $\lambda$ does not pass through $f$ and all vertices of $f$ lie on $\lambda$, then we call $f$ a $\lambda$-internal edge. See Figure 4 . For given $\lambda$ we denote the set of all $\lambda$-external (respectively $\lambda$-internal) edges by $\operatorname{Ex}_{\lambda}$ (respectively $\operatorname{In}_{\lambda}$ ).

By focusing vertices on (a component of) $\lambda$ and cutting $\lambda$-internal edges, one has the colored plane diagram as a part of $\Gamma \sqcup \lambda$ as in Figure 5. The diagram $D(\lambda, j)$ in the box consists of a combination of the two diagrams in Figure 6. (We take a convention for plane diagrams in which we draw the cycle $\lambda$ over the graph $\Gamma$.) Here thick lines in Figure 6 represent a part of $\lambda \subset \Gamma_{0}$ colored by $k$ and edges colored by $c$ correspond to $\lambda$ external/internal edges. We define a number $\epsilon_{\lambda}(v)$ for each trivalent vertex $v$ in $D(\lambda, j)$ as follows:

$$
\epsilon_{\lambda}(v)= \begin{cases}0 & \text { (if } \left.v \text { is type }(\mathrm{I})_{a, b, c}\right), \\ a-b & \left(\text { if } v \text { is type }(\mathrm{II})_{a, b, c}\right) .\end{cases}
$$

To describe the involution $Z(\lambda)$ explicitly, let us introduce the action of the homology of the graph on the set of $k$-admissible colorings. 


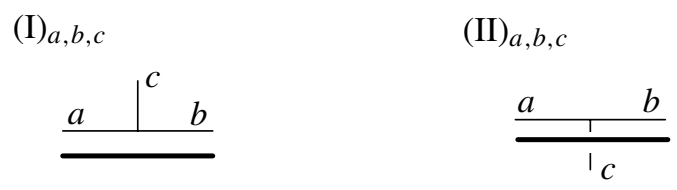

FIGURE 6. Diagrams in $D(\lambda, j)$.

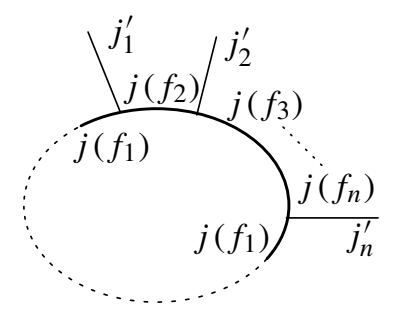

FIGURE 7. Colorings of $\lambda$-external/internal edges.

Definition 3.2. Let $\Gamma$ be a unitrivalent graph. For a $k$-admissible coloring $j$ of $\Gamma$ and a cycle $\lambda$ on $\Gamma$, define $\lambda \cdot j$ by

$$
\lambda \cdot j:=\left(\ldots, k-j_{l}, \ldots\right)
$$

that is, change all colors on the edges on $\lambda$ from $j_{l}$ to $k-j_{l}$, and the others are the same colors as those in $j$. One can check that $\lambda \cdot j$ is also a $k$-admissible coloring and this operation induces an action of $H_{1}(\Gamma ; \mathbb{Z} / 2)$ on the set of all $k$-admissible colorings of $\Gamma$.

Proposition 3.3. The involution $Z(\lambda)$ can be described as

$$
Z(\lambda):|j\rangle \mapsto \delta_{j}(\lambda)|\lambda \cdot j\rangle .
$$

Here $\delta_{j}$ is defined by

$$
\begin{aligned}
\delta_{j}(\lambda):=(-1)^{k n_{\lambda}+\sum j_{l}^{\prime} / 2} \prod_{f_{l} \subset \lambda} A^{\epsilon_{\lambda}(v)(k+2)} \frac{\left[k-j\left(f_{l}\right)\right] !}{\left[j\left(f_{l}\right)\right] !} \\
\times \frac{\left[\left(j\left(f_{l}\right)+j\left(f_{l+1}\right)+j_{l}^{\prime}\right) / 2+1\right] !}{\left[\left(k-j\left(f_{l}\right)+k-j\left(f_{l+1}\right)+j_{l}^{\prime}\right) / 2+1\right] !},
\end{aligned}
$$

where $n_{\lambda}$ is the number of edges on $\lambda, v$ runs over vertices on $\lambda$ and $j_{l}^{\prime}$ are colorings on $\lambda$-external or internal edges. (See Figure 7.)

Proof. Using formulas explained in the previous section we carry out the graphical computations for the diagrams (I) $)_{a, b, c}$ and (II) $a, b, c$. For the first we have the following.

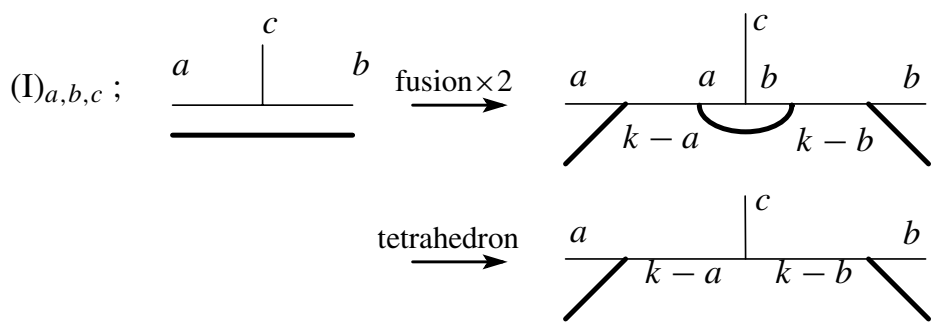


The coefficient associated with these diagrams is

$$
\begin{aligned}
& (\mathrm{I})_{a, b, c} ; \frac{\langle k-a\rangle}{\langle a k k-a\rangle} \frac{\langle k-b\rangle}{\langle b k k-b\rangle} \frac{\left\langle\begin{array}{ccc}
k-a & k-b & c \\
b & a & k
\end{array}\right|}{\langle k-a k-b c\rangle} \\
& =\langle k-a\rangle\langle k-b\rangle \frac{\left\langle\begin{array}{ccc}
k-a & k-b & c \\
b & a & k
\end{array}\right|}{\langle k-a k-b c\rangle} \\
& =\langle k-a\rangle\langle k-b\rangle(-1)^{(a+b-c) / 2} \frac{[k-a] ![k-b] ![(a+b+c) / 2+1] !}{[k+1] ![k+1-(a+b-c) / 2] !} .
\end{aligned}
$$

For the second we have the following.
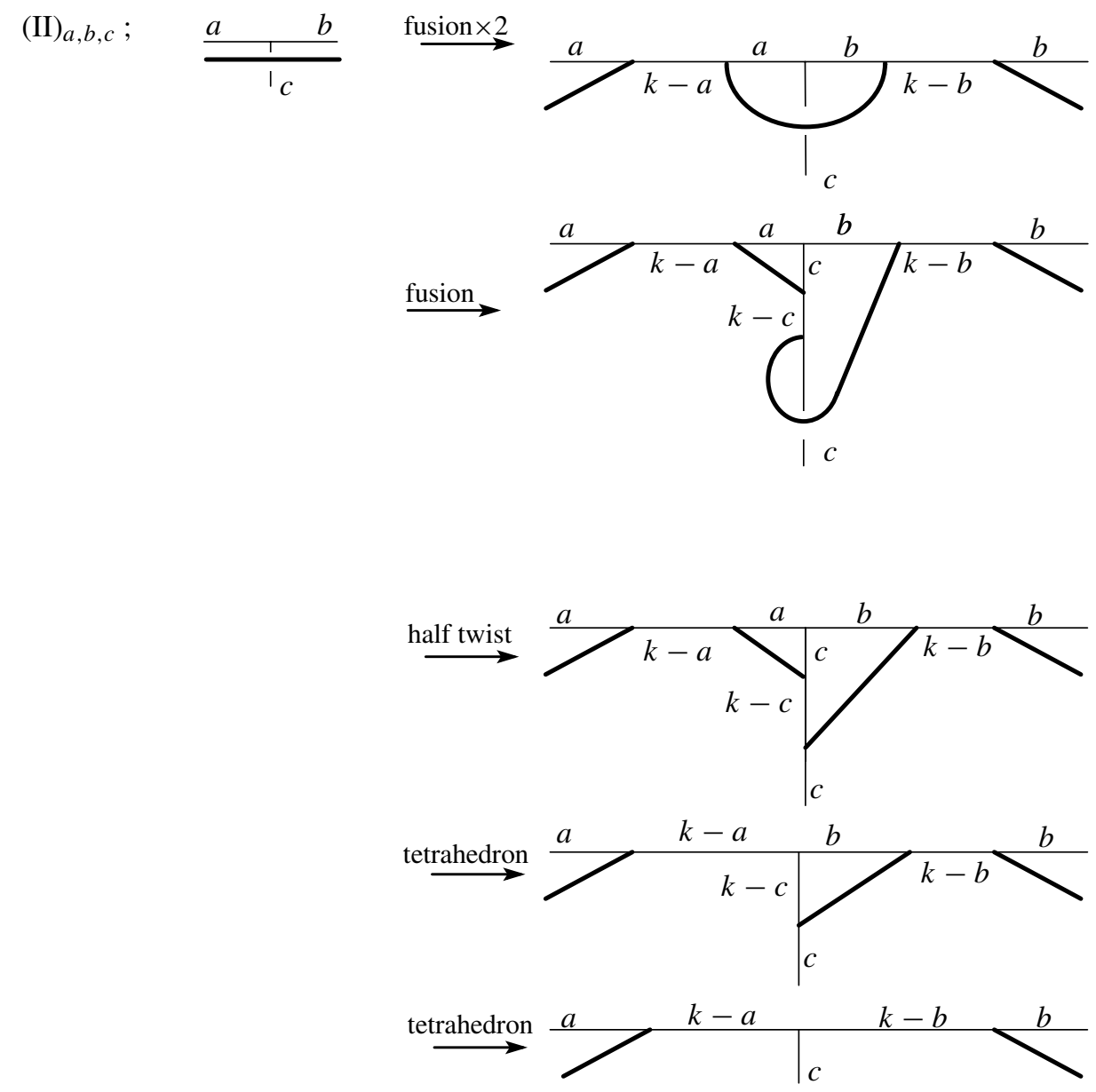

The coefficient associated with these diagrams is

$$
\text { (II) })_{a, b, c} ; \frac{\langle k-a\rangle}{\langle a k k-a\rangle} \frac{\langle k-b\rangle}{\langle b k k-b\rangle} \frac{\langle k-c\rangle}{\langle c k k-c\rangle} \overline{\delta(k-c ; k, c)}
$$




$$
\begin{aligned}
& \times \frac{\left(\begin{array}{ccc}
k-a & b & k-c \\
c & k & a
\end{array}\right)}{\langle k-a b k-c\rangle} \times \frac{\left(\begin{array}{ccc}
k-a & k-b & c \\
k & k-c & b
\end{array}\right)}{\langle k-a k-b c\rangle} \\
= & (-1)^{c} A^{c(k+2)}\langle k-a\rangle\langle k-b\rangle\langle k-c\rangle \\
& \times(-1)^{(a-b+c) / 2} \frac{[k-a] ![k-c] ![(a+b+c) / 2+1] !}{[k+1] ![k+1-(a-b+c) / 2] !} \\
& \times(-1)^{(a+b-c) / 2} \frac{[k-b] ![c] ![k+1-(a-b+c) / 2] !}{[k+1] ![k+1-(a+b-c) / 2] !} \\
= & A^{(2 c+c+a-b+c)(k+2)}\langle k-a\rangle\langle k-b\rangle(-1)^{(a+b-c) / 2} \\
& \times \frac{[k-a] ![k-b] ![(a+b+c) / 2+1] !}{[k+1] ![k+1-(a+b-c) / 2] !} \\
= & A^{(a-b)(k+2)} \times \text { coeff. of (I) } a, b, c .
\end{aligned}
$$

Here we note that $A^{2(k+2)}=-1$. By using these computations together with the equality (1) and

$$
[k-a] !^{2}=[k-a] ! \frac{[k+1] !}{[a+1] !}
$$

we have the explicit description of $\delta_{j}(\lambda)$ as in the proposition.

Remark 3.1. The computation given here was carried out by Masbaum a long time ago. Moreover he checked that by rescaling the basis the longitudes act by matrices whose entries are $\pm 1, \pm \sqrt{-1}$ or zero. Namely, put

$$
g(j):=\prod_{f: \text { edges of } \Gamma} \frac{1}{[j(f)] !} \prod_{v: \text { vertices of } \Gamma}[j(v)+1] !
$$

for a $k$-admissible coloring $j$, where $j(v)$ is defined by

$$
j(v):=\frac{j\left(f_{1}\right)+j\left(f_{2}\right)+j\left(f_{3}\right)}{2}
$$

for a vertex $v$ with edges $f_{1}, f_{2}$ and $f_{3}$. Then one has

$$
Z(\lambda): g(j)^{-1}|j\rangle \mapsto(-1)^{k n_{\lambda}+\sum j_{l}^{\prime} / 2} \prod_{f_{l} \subset \lambda} A^{\epsilon_{\lambda}(v)(k+2)} g(\lambda \cdot j)^{-1}|\lambda \cdot j\rangle .
$$

\subsection{External edge condition}

From the description of $Z(\lambda)$ obtained in the previous section, one can see that the trace of $Z(\lambda)$ is equal to the sum of coefficients $\delta_{j}(\lambda)$ for all $k$-admissible colorings $j$ with $\lambda \cdot j=j$. For such $\lambda$ and $j$, the diagram $D(\lambda, j)$ consists of diagrams (I) $k / 2, k / 2, c$ and (II) $k / 2, k / 2, c$. From now on we assume that $k$ is an even number because $k / 2$ should be a natural number $\dagger$. We also note that if $k$ is even and a triple $(k / 2, k / 2, c)$ is admissible then $c$ should be an even number. Then one can check the following proposition from the description of $\delta_{j}(\lambda)$.

PROPOSITION 3.4. The family of maps $\delta=\left(\delta_{j}\right)$ appearing in the representation matrices of $Z(\lambda)$ satisfies the external edge condition defined below.

$\dagger$ This fact implies that if $k$ is odd then the trace of $Z(\lambda)$ is equal to 0 . 
Definition 3.5. (External edge condition) Let $\alpha=\left(\alpha_{j}\right)$ be a family of maps parameterized by the set of $k$-admissible colorings of $\Gamma$ compatible with the given boundary coloring, where each map $\alpha_{j}$ is a map from $H_{1}(\Gamma ; \mathbb{Z} / 2)$ to the coefficient ring $R_{k}$ of $V_{2 k+4}$. We say that $\alpha$ satisfies the external edge condition if the following condition is satisfied:

$$
\alpha_{j}(\lambda)=(-1)^{\sum_{f_{l} \in \mathrm{Ex}_{\lambda}} j_{l} / 2} \quad \text { if } \lambda \cdot j=j .
$$

We only note that a $\lambda$-internal edge can be thought of as a $\lambda$-external edge with multiplicity two, and the coloring on such an edge contributes as $(-1)^{\text {even number }}=1$.

\section{Four lattices}

In this section we realize the first homology group of the surface in terms of a ribbon graph. Let $\Gamma$ be a unitrivalent ribbon graph with $3 g-3+2 n$ edges $E(\Gamma)=\left\{f_{l}\right\}, 2 g-2+n$ trivalent vertices and $n$ univalent vertices. We decompose the set of edges $E(\Gamma)$ into $E^{u}(\Gamma) \sqcup E^{t}(\Gamma)$, where $E^{u}(\Gamma)$ consists of edges with a univalent vertex and $E^{t}(\Gamma)$ consists of edges without a univalent vertex. Fix $3 g-3+2 n$ letters $\check{E}(\Gamma)=\left\{e_{l}\right\}$. These $e_{l}$ correspond to simple closed curves in the pants decomposition of $C_{\Gamma}^{\circ}$, the compact surface obtained by removing open disks around the banded link from $C_{\Gamma}$. We also have a decomposition $\check{E}(\Gamma)=\check{E}^{u}(\Gamma) \sqcup \check{E}^{t}(\Gamma)$. Here we put

$$
\left.\left.\check{E}^{u}(\Gamma) \text { (respectively } \check{E}^{t}(\Gamma)\right):=\left\{e_{l} \mid f_{l} \in E^{u}(\Gamma) \text { (respectively } E^{t}(\Gamma)\right)\right\} \text {. }
$$

Now we introduce four lattices $\Lambda_{0}, \Lambda, \Lambda_{0}^{*}$ and $\Lambda^{*}$ as follows $\dagger$.

Definition 4.1. Let $\mathbb{R}\langle\check{E}(\Gamma)\rangle$ be the vector space generated by $\check{E}(\Gamma)=\left\{e_{l}\right\}$ over $\mathbb{R}$. Let $\Lambda_{0}:=\mathbb{Z}\langle\check{E}(\Gamma)\rangle$ be the standard lattice in $\mathbb{R}\langle\check{E}(\Gamma)\rangle$ and $\Lambda$ be the lattice generated by the elements $\left\{E_{1}^{i}, E_{2}^{i}, E_{3}^{i} \mid i=1, \ldots, 2 g-2+n\right\}$, where these elements are defined as follows. For each trivalent vertex $v_{i}$ with edges $f_{i_{1}}, f_{i_{2}}$ and $f_{i_{3}}$ define $E_{1}^{i}, E_{2}^{i}$ and $E_{3}^{i}$ by

$$
\begin{aligned}
& E_{1}^{i}=\frac{1}{2}\left(-e_{i_{1}}+e_{i_{2}}+e_{i_{3}}\right), \\
& E_{2}^{i}=\frac{1}{2}\left(e_{i_{1}}-e_{i_{2}}+e_{i_{3}}\right), \\
& E_{3}^{i}=\frac{1}{2}\left(e_{i_{1}}+e_{i_{2}}-e_{i_{3}}\right) .
\end{aligned}
$$

If a vertex $v_{i}$ has only two edges $f_{i_{1}}$ and $f_{i_{2}}=f_{i_{3}}$ then we interpret as

$$
\begin{aligned}
& E_{1}^{i}:=-\frac{1}{2} e_{i_{1}}+e_{i_{2}}, \\
& E_{2}^{i}=E_{3}^{i}:=\frac{1}{2} e_{i_{1}} .
\end{aligned}
$$

Let $\mathbb{R}\left\langle E^{t}(\Gamma)\right\rangle$ be the $\mathbb{R}$-vector space generated by $E^{t}(\Gamma)$. Let $\Lambda_{0}^{*}=\mathbb{Z}\left\langle E^{t}(\Gamma)\right\rangle$ be the standard lattice in $\mathbb{R}\left\langle E^{t}(\Gamma)\right\rangle$ and $\Lambda^{*}$ be the sublattice of $\Lambda_{0}^{*}$ defined by

$$
\Lambda^{*}:=\left\{\sum_{l} n_{l} f_{l} \in \Lambda_{0}^{*} \mid n_{i_{1}}+n_{i_{2}}+n_{i_{3}} \in 2 \mathbb{Z}\right\} .
$$

There is a natural pairing between $\mathbb{R}\langle\check{E}(\Gamma)\rangle$ and $\mathbb{R}\left\langle E^{t}(\Gamma)\right\rangle$ denoted by $\cdot$, which is defined by $e_{l} \cdot f_{l^{\prime}}=\delta_{l, l^{\prime}}$. Note that this pairing is non-degenerate except for the subspace generated by $\check{E}^{u}(\Gamma)$.

$\dagger$ These lattices are introduced in [8] for a closed surface with a pants decomposition. 


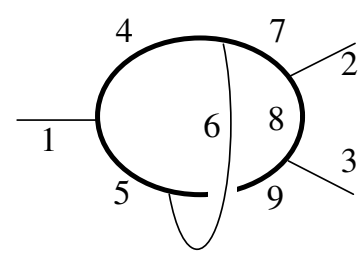

FIGURE 8 . The thick line corresponds to $\lambda=f_{4}+f_{5}+f_{7}+f_{8}+f_{9}$.

Remark 4.1. Note that $\Lambda$ is a sublattice of $\Lambda_{0}$ with $\Lambda / \Lambda_{0} \cong(\mathbb{Z} / 2)^{2 g-2+2 n-n^{\prime}}$, where $n^{\prime}=n(n \geq 1), 1(n=0)$. This follows from the facts that $E_{1}^{i} \equiv E_{2}^{i} \equiv E_{3}^{i} \bmod \Lambda_{0}$ for $i=$ $1, \ldots, 2 g-2+n$ and $\sum_{i} E_{1}^{i} \equiv 0 \bmod \Lambda_{0}$ if $n=0$.

PROPOSITION 4.2. We have the following:

(1) there is a canonical isomorphism $\Lambda^{*} / 2 \Lambda_{0}^{*} \cong H_{1}(\Gamma ; \mathbb{Z} / 2)\left(\cong(\mathbb{Z} / 2)^{g}\right)$;

(2) the inclusion $\Gamma_{0} \subset C_{\Gamma}$ induces a decomposition $H_{1}\left(C_{\Gamma}^{\circ} ; \mathbb{Z} / 2\right) \cong \Lambda_{0} / 2 \Lambda \oplus \Lambda^{*} / 2 \Lambda_{0}^{*}$.

Proof. (1) The required isomorphism is given as follows. By definition each $\lambda \in \Lambda^{*} / 2 \Lambda_{0}^{*}$ can be represented by an element in $\Lambda^{*}$ which has two (or no) non-trivial entries at each trivalent vertex. Putting edges corresponding to non-trivial entries in $\lambda$ together we obtain a cycle in $\Gamma$. Conversely for a given cycle in $\Gamma$ we have an element in $\Lambda^{*} / 2 \Lambda_{0}^{*}$ whose non-trivial entries correspond with edges on the cycle. See Figure 8.

(2) The elements in $2 \Lambda$ correspond to boundaries of the pants decomposition of the surface $C_{\Gamma}^{\circ}$ up to orientation. Hence there is a natural map $\Lambda_{0} / 2 \Lambda \rightarrow H_{1}\left(C_{\Gamma}^{\circ} ; \mathbb{Z} / 2\right)$, and one can check that this is an inclusion. Then the rank of the cokernel of this inclusion is equal to $g$ because we have that $\Lambda_{0} / 2 \Lambda \cong(\mathbb{Z} / 2)^{g+n^{\prime}-1}$. On the other hand we have a natural map $\Lambda^{*} / 2 \Lambda_{0}^{*} \cong H_{1}\left(\Gamma_{0} ; \mathbb{Z} / 2\right) \rightarrow H_{1}\left(C_{\Gamma}^{\circ} ; \mathbb{Z} / 2\right)$ via the inclusion $\Gamma_{0} \subset C_{\Gamma}$. It induces an injection $\Lambda^{*} / 2 \Lambda_{0}^{*} \rightarrow H_{1}\left(C_{\Gamma}^{\circ} ; \mathbb{Z} / 2\right) /\left(\Lambda_{0} / 2 \Lambda\right)$, and this is an isomorphism because these have the same rank $g$. This construction gives a splitting of the exact sequence $0 \rightarrow \Lambda_{0} / 2 \Lambda \rightarrow$ $H_{1}\left(C_{\Gamma}^{\circ} ; \mathbb{Z} / 2\right) \rightarrow H_{1}\left(C_{\Gamma}^{\circ} ; \mathbb{Z} / 2\right) /\left(\Lambda_{0} / 2 \Lambda\right) \cong \Lambda^{*} / 2 \Lambda_{0}^{*} \rightarrow 0$.

Remark 4.2. Note that the subgroup $\Lambda_{0}^{u} / 2 \Lambda^{u}$ is isomorphic to the subgroup of $H_{1}\left(C_{\Gamma}^{\circ} ; \mathbb{Z} / 2\right)$ generated by boundaries of $C_{\Gamma}^{\circ}$, where $\Lambda_{0}^{u}$ is the lattice generated by $\check{E}^{u}(\Gamma)$ and we put $2 \Lambda^{u}:=\Lambda_{0}^{u} \cap 2 \Lambda$.

Remark 4.3. The isomorphism $H_{1}\left(C_{\Gamma}^{\circ} ; \mathbb{Z} / 2\right) \cong \Lambda_{0} / 2 \Lambda \oplus \Lambda^{*} / 2 \Lambda_{0}^{*}$ is compatible with the intersection pairing. Namely, the mod 2 intersection pairing between meridians and longitudes coincides with the natural pairing between $\Lambda_{0} / 2 \Lambda$ and $\Lambda^{*} / 2 \Lambda_{0}^{*}$ induced from the pairing between $\Lambda_{0}$ and $\Lambda_{0}^{*}$. We denote this pairing by $\cdot: \Lambda_{0} / 2 \Lambda \oplus \Lambda^{*} / 2 \Lambda_{0}^{*} \rightarrow \mathbb{Z} / 2$, $(\mu, \lambda) \mapsto \mu \cdot \lambda$.

\section{Representation matrix of the Heisenberg action}

In this section we describe the Heisenberg action in [5] explicitly in terms of a ribbon graph and its admissible colorings. We first review the construction of the Heisenberg type group in [5]. For an oriented surface $C$, let $H(C)$ be the direct product of $\mathbb{Z}$ and 
$H_{1}(C ; \mathbb{Z})$ with multiplication given by $(n, x)(m, y):=(n+m+x \cdot y, x+y)$, where $x \cdot y$ is the intersection number of $x$ and $y$. We denote $(n, 0)$ by $u^{n}$ for $n \in \mathbb{Z}$ and $[x]=(0, x)$ for $x \in H_{1}(C ; \mathbb{Z})$.

Definition 5.1. Let $\mathcal{E}(C)$ be the quotient of $H(C)$ by the subgroup generated by $u^{4}$ and elements $[2 x]$. One can check that $\mathcal{E}(C)$ is a central extension

$$
\mathbb{Z} / 4 \rightarrow \mathcal{E}(C) \rightarrow H_{1}(C ; \mathbb{Z} / 2)
$$

For the surface $C_{\Gamma}$ associated with a ribbon graph $\Gamma$, we denote $\mathcal{E}\left(C_{\Gamma}\right)$ by $\mathcal{E}(\Gamma)$. We can define a map $\tau: \Lambda_{0} / 2 \Lambda \oplus \Lambda^{*} / 2 \Lambda_{0}^{*} \rightarrow \mathcal{E}(\Gamma)$ as follows. We first note that $\mu \in \Lambda_{0} / 2 \Lambda$ is represented by a disjoint union of simple closed curves $\tilde{\mu}$ on $C_{\Gamma}$, and it determines an element in $H_{1}\left(C_{\Gamma} ; \mathbb{Z}\right)$ up to sign. The induced element $\tau(\mu):=[\tilde{\mu}] \in \mathcal{E}(\Gamma)$ depends only on $\mu$ since $\tilde{\mu} \cdot \tilde{\mu}=0$ and hence $[\tilde{\mu}]=[-\tilde{\mu}]$. Similarly we have an element $\tau(\lambda) \in \mathcal{E}(\Gamma)$. We define $\tau(\mu, \lambda):=u^{\mu \circ \lambda} \tau(\lambda) \tau(\mu)$, where $\mu \circ \lambda$ is the geometric intersection number of $\mu$ and $\lambda$.

Remark 5.1. Put $\mathcal{E}^{\prime}(\Gamma):=\mathbb{Z} / 2 \times\left(\Lambda_{0} / 2 \Lambda \oplus \Lambda^{*} / 2 \Lambda_{0}^{*}\right)$ as a set. Define a group structure on $\mathcal{E}^{\prime}(\Gamma)$ by

$$
\left(c_{1}, \mu_{1}, \lambda_{1}\right) \cdot\left(c_{2}, \mu, \lambda_{2}\right)=\left(c_{1} c_{2}(-1)^{\lambda_{2} \cdot \mu_{1}}, \mu_{1}+\mu_{2}, \lambda_{1}+\lambda_{2}\right),
$$

where is the natural pairing between $\Lambda_{0} / 2 \Lambda$ and $\Lambda^{*} / 2 \Lambda_{0}^{*}$. Then $\mathcal{E}^{\prime}(\Gamma)$ is a central extension

$$
\mathbb{Z} / 2 \rightarrow \mathcal{E}^{\prime}(\Gamma) \rightarrow \Lambda_{0} / 2 \Lambda \oplus \Lambda^{*} / 2 \Lambda_{0}^{*}
$$

Then one can check that $\mathcal{E}^{\prime}(\Gamma)$ is a reduction of $\mathcal{E}(\Gamma)$ to a $\mathbb{Z} / 2$-extension. Namely the $\mathbb{Z} / 4$ extension induced from $\mathcal{E}^{\prime}(\Gamma)$ via the natural inclusion $\mathbb{Z} / 2 \hookrightarrow \mathbb{Z} / 4$ is isomorphic to $\mathcal{E}(\Gamma)$.

Fix a non-negative integer $k$ and a boundary coloring $j^{\prime}=\left(j_{l}^{\prime}\right)$. We have the TQFTmodule $V_{2 k+4}\left(C_{\Gamma}\right)=V_{2 k+4}\left(C_{\Gamma} ; j^{\prime}\right)$, which has a basis parameterized by the finite set of all $k$-admissible colorings. By previous computations, we have the following descriptions of the involutions of meridian cycles and longitude cycles.

Meridian. For $\mu \in \Lambda_{0} / 2 \Lambda$ and a $k$-admissible coloring $j$ one has

$$
\tau(\mu):|j\rangle \mapsto(-1)^{j_{\mu}}|j\rangle,
$$

where $j_{\mu}$ is defined by $j_{\mu}:=\sum_{\epsilon_{l} \neq 0} j_{l}$ for $\mu=\sum_{l} \epsilon_{l} e_{l}$.

Longitude. For $\lambda \in \Lambda^{*} / 2 \Lambda_{0}^{*}$ and a $k$-admissible coloring $j$ one has

$$
\tau(\lambda):|j\rangle \mapsto \delta_{j}(\lambda)|\lambda \cdot j\rangle,
$$

where the coefficient $\delta_{j}(\lambda)$ can be computed as in the previous section and it satisfies the external edge condition (Definition 3.5).

Combining these descriptions and the action of central elements (see [5, Proposition 7.5]) we have the following description of $\mathcal{E}(\Gamma)$-action on $V_{2 k+4}\left(C_{\Gamma}\right)$ :

$$
\begin{aligned}
& \rho^{(k)}: \mathcal{E}(\Gamma) \rightarrow G L\left(V_{2 k+4}\left(C_{\Gamma}\right)\right), \\
& u^{m} \tau(\mu, \lambda) \mapsto\left(|j\rangle \mapsto A^{(k+2)^{2}(m+\mu \circ \lambda)}(-1)^{(k+1)(m+\mu \circ \lambda)+j_{\mu}} \delta_{j}(\lambda)|\lambda \cdot j\rangle\right) .
\end{aligned}
$$


Remark 5.2. If $k$ is an even number then one has that

$$
\rho^{(k)}(\tau(a)) \rho^{(k)}(\tau(b))=(-1)^{(k / 2) a \cdot b} \rho^{(k)}(\tau(a+b))
$$

by direct computation. In particular, in the case of even $k$, the representation $\rho^{(k)}$ is an abelian.

\section{Trace of the Heisenberg action}

In this section we compute the trace of the involutions on the TQFT-module by making use of the previous results. The formula for closed surfaces with an empty link is obtained in [2] and [5]. Our computation works uniformly for surfaces with a non-empty link, and the formula is the same as the formula for a surface without a banded link up to some factor $(\in\{-1,0,1\})$ that is determined by the boundary coloring.

Let $\Gamma$ be a ribbon unitrivalent graph. Fix a boundary coloring $j^{\prime}$. For a non-negative integer $k$ we denote by $Q C G_{k}\left(\Gamma ; j^{\prime}\right)$ the set of all $k$-admissible colorings of $\Gamma$ compatible with the given boundary coloring $j^{\prime}$. By the description of $\mathcal{E}(\Gamma)$-action, the trace of $\rho^{(k)}(\tau(\mu, \lambda))$ is given by

$$
\operatorname{Tr}\left(\rho^{(k)}(\tau(\mu, \lambda)) ; V_{2 k+4}\left(C_{\Gamma} ; j^{\prime}\right)\right)=\sum_{j \in Q C G_{k}^{\lambda}\left(\Gamma ; j^{\prime}\right)} A^{(k+2)^{2} \mu \circ \lambda}(-1)^{(k+1) \mu \circ \lambda+j_{\mu}} \delta_{j}(\lambda),
$$

where $Q C G_{k}^{\lambda}\left(\Gamma ; j^{\prime}\right)$ is the set of all $k$-admissible colorings fixed by the action of $\lambda$;

$$
Q C G_{k}^{\lambda}\left(\Gamma ; j^{\prime}\right):=\left\{j \in Q C G_{k}\left(\Gamma ; j^{\prime}\right) \mid \lambda \cdot j=j\right\} .
$$

In this section we compute the right-hand side directly and show the following formula.

THEOREM 6.1. We have the following trace formula:

$$
\operatorname{Tr}\left(\rho^{(k)}(\tau(\mu, \lambda)) ; V_{2 k+4}\left(C_{\Gamma} ; j^{\prime}\right)\right)=\gamma\left(j^{\prime}\right) \frac{1+(-1)^{k}}{2}\left(\frac{k+2}{2}\right)^{g-1}
$$

for $\mu \in \Lambda_{0} / 2 \Lambda$ and $\lambda(\neq 0) \in \Lambda^{*} / 2 \Lambda_{0}^{*}$ or for $\mu \notin \Lambda_{0}^{u} / 2 \Lambda^{u}$ and $\lambda=0$, where $\gamma\left(j^{\prime}\right) \in$ $\{-1,0,1\}$ is defined below (Definitions/Notation (3)). For $\mu=\sum \epsilon_{l} e_{l} \in \Lambda_{0}^{u} / 2 \Lambda^{u}$, one has $\operatorname{Tr}\left(\rho^{(k)}(\mu, 0) ; V_{2 k+4}\left(C_{\Gamma} ; j^{\prime}\right)\right)=(-1)^{\sum \epsilon_{l} j_{l}^{\prime}{ }^{\#}} Q C G_{k}\left(\Gamma ; j^{\prime}\right)$.

The last part follows from the description of the action of a cycle around a component of the banded link. The case for odd $k$ follows from the following lemma.

LEMMA 6.2. If $k$ is an odd number, then we have $\operatorname{Tr}\left(\rho^{(k)}(\tau(\mu, \lambda)) ; V_{2 k+4}\left(C_{\Gamma} ; j^{\prime}\right)\right)=0$ for $\mu \in \Lambda_{0} / 2 \Lambda$ and $\lambda(\neq 0) \in \Lambda^{*} / 2 \Lambda_{0}^{*}$ or $\mu \notin \Lambda_{0}^{u} / 2 \Lambda^{u}$ and $\lambda=0$.

Proof. First note that if $\lambda \cdot j=j$ then $j_{l}=k / 2$ for all $l$ with $\lambda_{l} \neq 0$. But $k / 2$ is not an integer if $k$ is odd. This implies that for an odd number $k$ and $\lambda \neq 0$ we have $Q C G_{k}^{\lambda}\left(\Gamma ; j^{\prime}\right)=\emptyset$, and hence $\operatorname{Tr}\left(\rho^{(k)}(\mu, \lambda) ; V_{2 k+4}\left(C_{\Gamma} ; j^{\prime}\right)\right)=0$. Now we assume that $k$ is an odd number and $\lambda=0, \mu \notin \Lambda_{0}^{u} / 2 \Lambda^{u}$. In this case we have

$$
\begin{aligned}
\operatorname{Tr}\left(\rho^{(k)}(\mu, 0) ; V_{2 k+4}\left(C_{\Gamma} ; j^{\prime}\right)\right) & =\sum_{j \in Q C G_{k}^{\lambda}\left(\Gamma ; j^{\prime}\right)}(-1)^{j_{\mu}} \\
& ={ }^{\#}\left\{j \in Q C G_{k} \mid j_{\mu} \in 2 \mathbb{Z}\right\}-{ }^{\#}\left\{j \in Q C G_{k} \mid j_{\mu} \notin 2 \mathbb{Z}\right\} .
\end{aligned}
$$


Take and fix a cycle $\lambda_{\mu} \in \Lambda^{*} / 2 \Lambda_{0}^{*}$ on $\Gamma$ with $\mu \cdot \lambda_{\mu} \equiv 1 \bmod 2$. Then a map

$$
\begin{aligned}
\left\{j \in Q C G_{k} \mid j_{\mu} \in 2 \mathbb{Z}\right\} & \longleftrightarrow\left\{j \in Q C G_{k} \mid j_{\mu} \notin 2 \mathbb{Z}\right\}, \\
j & \longleftrightarrow \lambda_{\mu} \cdot j
\end{aligned}
$$

gives a bijection if $k$ is an odd number because of the relation

$$
\left(\lambda_{\mu} \cdot j\right)_{\mu}=k \lambda_{\mu} \cdot \mu+j_{\mu} \bmod \mathbb{Z} .
$$

In particular we obtain $\operatorname{Tr}\left(\rho^{(k)}(\mu, 0) ; V_{2 k+4}\left(C_{\Gamma} ; j^{\prime}\right)\right)=0$.

Now we make several preparations to show the theorem for even $k$. Hereafter we assume that $k$ is an even natural number. We introduce some definitions and notation (see also Example 6.3).

\section{Definitions/Notation.}

(1) For given $(\mu, \lambda) \in \Lambda_{0} / 2 \Lambda \oplus \Lambda^{*} / 2 \Lambda_{0}^{*}$ take and fix representatives $\mu=\sum_{l} \epsilon_{l} e_{l}$ and $\lambda=\sum_{l} \epsilon_{l}^{\prime} f_{l}$ (we denote them by the same letters), and define $\mu(\lambda)$ by $\mu(\lambda):=$ $\sum_{l} \epsilon_{l}\left(1-\epsilon_{l}^{\prime}\right) e_{l}$. We consider the decomposition of the graph $\Gamma$ into $\Gamma(\lambda) \cup \Gamma^{\prime}(\lambda)$ and $\Gamma(\lambda) \cup \Gamma^{\prime}(\lambda)_{\mu}$ as follows:

$\Gamma(\lambda):=\left\{f_{l}, v_{i}, w_{i} \mid f_{l}\right.$ with $\lambda_{l} \neq 0$ or $f_{l} \in \operatorname{Ex}_{\lambda} \cup \operatorname{In}_{\lambda}$, and $v_{i}$ or $w_{i}$ is a vertex of $\left.f_{l}\right\}$;

$\Gamma^{\prime}(\lambda):=\left\{f_{l}, v_{i}, w_{i} \mid f_{l}\right.$ with $\lambda_{l}=0$ or $f_{l} \in \operatorname{Ex}_{\lambda}$, and $v_{i}$ or $w_{i}$ is a vertex of $\left.f_{l}\right\}$;

$\Gamma^{\prime}(\lambda)_{\mu}$; the graph obtained by cutting the edges in $\Gamma^{\prime}(\lambda)$ with $\mu(\lambda)_{l} \neq 0$.

(2) For a given graph $\cdot$, we denote by $E^{u}(\cdot)$ the set of edges that have one univalent vertex and by $E^{t}(\cdot)$ the set of edges that have no univalent vertex. Note that $E^{u}(\Gamma(\lambda))=\operatorname{Ex}_{\lambda}$. We define the subset of edges as follows:

$$
\begin{aligned}
& E_{\lambda}^{u}:=\operatorname{Ex}_{\lambda} \cap E^{u}(\Gamma) \subset E^{u}(\Gamma(\lambda)) ; \\
& E_{\lambda}^{t}:=\operatorname{Ex}_{\lambda} \cap E^{t}(\Gamma) \subset E^{u}(\Gamma(\lambda)) ; \\
& \overline{E_{\lambda}^{u}}:=E^{u}(\Gamma)-E_{\lambda}^{u} \subset E^{u}\left(\Gamma^{\prime}(\lambda)\right) ; \\
& E_{\mu}^{u}:=E^{u}\left(\Gamma^{\prime}(\lambda)_{\mu}\right)-\left(\operatorname{Ex}_{\lambda} \cup \overline{E_{\lambda}^{u}}\right) \subset E^{u}\left(\Gamma^{\prime}(\lambda)_{\mu}\right) .
\end{aligned}
$$

(3) For a subset $E^{\prime}$ of $E^{u}(\cdot)$ for a given graph $\cdot$, we denote by $j^{\prime}\left(E^{\prime}\right)$ the set of given boundary colorings of $E^{\prime}$, and we put $\sum j^{\prime}\left(E^{\prime}\right):=\sum_{f_{l} \in E^{\prime}} j_{l}^{\prime}$. We also define $\gamma\left(j^{\prime}\left(E^{\prime}\right)\right) \in\{-1,0,1\}$ by

$$
\gamma\left(j^{\prime}\left(E^{\prime}\right)\right):= \begin{cases}(-1)^{\sum j^{\prime}\left(E^{\prime}\right) / 2} & \left(\forall f_{l} \in E^{\prime}, \quad j_{l}^{\prime} \in 2 \mathbb{Z}\right), \\ 0 & \left(\exists f_{l} \in E^{\prime}, \quad j_{l}^{\prime} \notin 2 \mathbb{Z}\right) .\end{cases}
$$

(4) We put $n_{1}:={ }^{\#} E_{\lambda}^{u}, n_{2}:={ }^{\#} E_{\lambda}^{u}$ and we assume that $\Gamma(\lambda)$ has $3 g_{1}-3+2\left(n_{1}+m\right)$ edges and $\Gamma^{\prime}(\lambda)$ has $3 g_{2}-3+2\left(n_{2}+m\right)$ edges (then $g=g_{1}+g_{2}+m-1$ and $m=$ $\left.{ }^{\#} E_{\lambda}^{t}\right)$. Note that $E_{\mu}^{u}$ has an even element, and we put $2 m^{\prime}:={ }^{\#} E_{\mu}^{u}$.

(5) Let $Q C G_{k}^{\lambda}\left(\Gamma ; j^{\prime}(\Gamma)\right)$ be the subset of all admissible colorings for $\left(\Gamma ; j^{\prime}(\Gamma)\right)$ fixed by the action of $\lambda$, i.e.

$$
\operatorname{QCG}_{k}^{\lambda}\left(\Gamma ; j^{\prime}(\Gamma)\right)=\left\{j \in Q C G_{k}\left(\Gamma ; j^{\prime}(\Gamma)\right) \mid j_{l}=k / 2 \text { if } \lambda_{l} \neq 0\right\} .
$$

Similarly we can define the subset $Q C G_{k}^{\lambda}\left(\Gamma(\lambda) ; j^{\prime}(\Gamma(\lambda))\right) \subset Q C G_{k}\left(\Gamma(\lambda) ; j^{\prime}(\Gamma(\lambda))\right)$. 


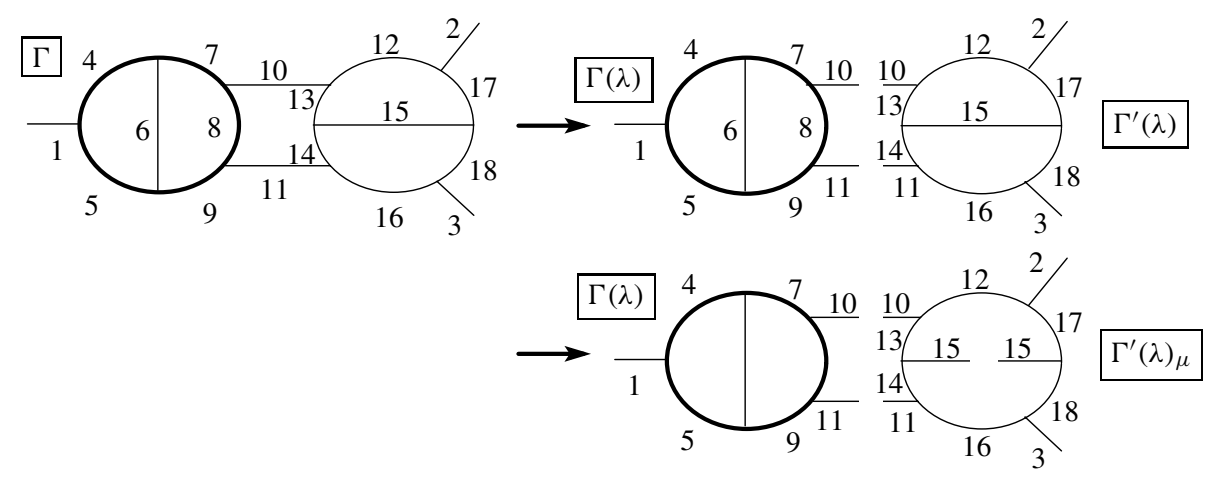

FigurE 9. Examples of $\Gamma(\lambda), \Gamma^{\prime}(\lambda)$ and $\Gamma^{\prime}(\lambda) \mu$.

Example 6.3. An example of $\Gamma(\lambda), \Gamma^{\prime}(\lambda)$ and $\Gamma^{\prime}(\lambda)_{\mu}$ is shown in Figure 9. We take $\mu=$ $e_{6}+e_{8}+e_{15}$ and $\lambda=f_{4}+f_{5}+f_{7}+f_{8}+f_{9}$. In this case we have $\mu(\lambda)=e_{15}, E_{\lambda}^{u}=\left\{f_{1}\right\}$, $E_{\lambda}^{t}=\left\{f_{10}, f_{11}\right\}, \overline{E_{\lambda}^{u}}=\left\{f_{2}, f_{3}\right\}$ and $E_{\mu}^{u}=\left\{f_{15}, f_{15}\right\}$.

Theorem 6.1 is shown by the following two propositions.

PROPOSITION 6.4. We have the equality

$$
{ }^{\#} Q C G_{k}^{\lambda}\left(\Gamma(\lambda) ; j^{\prime}(\Gamma(\lambda))\right)=\gamma\left(j^{\prime}\left(\operatorname{Ex}_{\lambda}\right)\right)^{2}\left(\frac{\mathrm{k}+2}{2}\right)^{\mathrm{g}_{1}-1} .
$$

PROPOSITION 6.5. We have the equality

$$
\begin{aligned}
& \sum_{j^{\prime}\left(E_{\lambda}^{t}\right), j^{\prime}\left(E_{\mu}^{u}\right)}(-1)^{\sum j^{\prime}\left(E_{\lambda}^{t}\right) / 2+\sum j^{\prime}\left(E_{\mu}^{u}\right) \#} Q C G_{k}\left(\Gamma^{\prime}(\lambda)_{\mu} ; j^{\prime}\left(\Gamma^{\prime}(\lambda)_{\mu}\right)\right) \\
& =\gamma\left(j^{\prime}\left(\overline{E_{\lambda}^{u}}\right)\right)\left(\frac{k+2}{2}\right)^{g_{2}-1+m} \cdot
\end{aligned}
$$

By using these propositions we can prove Theorem 6.1 by an elementary counting argument.

Proof of Theorem 6.1. Note that for an even number $k$ one has $A^{(k+2)^{2} \mu \circ \lambda}(-1)^{(k+1) \mu \circ \lambda+j_{\mu}}=$ $(-1)^{(k / 2) \mu \cdot \lambda+j_{\mu}}$. By using this equality and the fact that $\delta_{j}$ satisfies the external edge condition, the trace can be computed in the following way:

$$
\begin{aligned}
& \sum_{j \in Q C G_{k}^{\lambda}\left(\Gamma ; j^{\prime}(\Gamma)\right)}(-1)^{(k / 2) \mu \cdot \lambda+j_{\mu}} \delta_{j}(\lambda) \\
= & \sum_{j \in Q C G_{k}^{\lambda}\left(\Gamma ; j^{\prime}(\Gamma)\right)}(-1)^{(k / 2) \mu \cdot \lambda+j_{\mu}+\sum_{f_{l} \in \mathrm{Ex}_{\lambda}} j_{l} / 2} \\
= & (-1)^{(k / 2) \mu \cdot \lambda}\left(\#\left\{j \in Q C G_{k}^{\lambda} \mid j_{\mu}+\sum_{f_{l} \in \operatorname{Ex}_{\lambda}} \frac{j_{l}}{2} \equiv 0 \bmod 2\right\}\right. \\
& \left.-\#\left\{j \in Q C G_{k}^{\lambda} \mid j_{\mu}+\sum_{f_{l} \in \operatorname{Ex}_{\lambda}} \frac{j_{l}}{2} \equiv 1 \bmod 2\right\}\right)
\end{aligned}
$$




$$
\begin{aligned}
& =(-1)^{(k / 2) \mu \cdot \lambda}\left(\#\left\{j \in Q C G_{k}^{\lambda} \mid j_{\mu(\lambda)}+\frac{k}{2} \mu \cdot \lambda+\sum_{f_{l} \in \mathrm{Ex}_{\lambda}} \frac{j_{l}}{2} \equiv 0 \bmod 2\right\}\right. \\
& \left.-\#\left\{j \in Q C G_{k}^{\lambda} \mid j_{\mu(\lambda)}+\frac{k}{2} \mu \cdot \lambda+\sum_{f_{l} \in \mathrm{Ex}_{\lambda}} \frac{j_{l}}{2} \equiv 1 \bmod 2\right\}\right) \\
& =\#\left\{j \in Q C G_{k}^{\lambda} \mid j_{\mu(\lambda)}+\sum_{f_{l} \in \mathrm{Ex}_{\lambda}} \frac{j_{l}}{2} \equiv 0 \bmod 2\right\} \\
& -\#\left\{j \in Q C G_{k}^{\lambda} \mid j_{\mu(\lambda)}+\sum_{f_{l} \in \mathrm{Ex}_{\lambda}} \frac{j_{l}}{2} \equiv 1 \bmod 2\right\} \\
& =\#\left\{j \in Q C G_{k}^{\lambda} \mid j_{\mu(\lambda)}, \sum_{f_{l} \in \mathrm{Ex}_{\lambda}} \frac{j_{l}}{2} \in 2 \mathbb{Z}\right\}-\#\left\{j \in Q C G_{k}^{\lambda} \mid j_{\mu(\lambda)} \in 2 \mathbb{Z}, \sum_{f_{l} \in \mathrm{Ex}_{\lambda}} \frac{j_{l}}{2} \notin 2 \mathbb{Z}\right\} \\
& +\#\left\{j \in Q C G_{k}^{\lambda} \mid j_{\mu(\lambda)} \notin 2 \mathbb{Z}, \sum_{f_{l} \in \mathrm{Ex}_{\lambda}} \frac{j_{l}}{2} \notin 2 \mathbb{Z}\right\}
\end{aligned}
$$

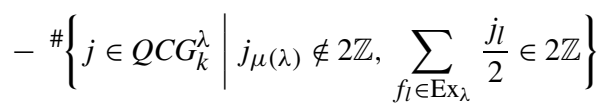

$$
\begin{aligned}
& =\sum_{j^{\prime}\left(\operatorname{Ex}_{\lambda}\right)}(-1)^{\sum j^{\prime}\left(\operatorname{Ex}_{\lambda}\right) / 2{ }^{\#}} Q C G_{k}^{\lambda}\left(\Gamma(\lambda) ; j^{\prime}(\Gamma(\lambda))\right){ }^{\#}\left\{j \in Q C G_{k}\left(\Gamma^{\prime}(\lambda) ; j^{\prime}\left(\Gamma^{\prime}(\lambda)\right)\right) \mid j_{\mu(\lambda)} \in 2 \mathbb{Z}\right\} \\
& -\sum_{j^{\prime}\left(\operatorname{Ex}_{\lambda}\right)}(-1)^{\sum j^{\prime}\left(\operatorname{Ex}_{\lambda}\right) / 2 \#} Q C G_{k}^{\lambda}\left(\Gamma(\lambda) ; j^{\prime}(\Gamma(\lambda))\right){ }^{\#}\left\{j \in Q C G_{k}\left(\Gamma^{\prime}(\lambda) ; j^{\prime}\left(\Gamma^{\prime}(\lambda)\right)\right) \mid j_{\mu(\lambda)} \notin 2 \mathbb{Z}\right\} \\
& =\left(\frac{k+2}{2}\right)^{g_{1}-1} \prod_{f_{l} \in E_{\lambda}^{u}} \sin ^{2+1} \frac{j_{l}^{\prime}+1}{2} \pi \sum_{j^{\prime}\left(E_{\lambda}^{t}\right)}(-1)^{\sum j^{\prime}\left(E_{\lambda}^{t}\right) / 2} \\
& \times\left({ }^{\#}\left\{j \in Q C G_{k}\left(\Gamma^{\prime}(\lambda) ; j^{\prime}\left(\Gamma^{\prime}(\lambda)\right)\right) \mid j_{\mu(\lambda)} \in 2 \mathbb{Z}\right\}\right. \\
& \text { - } \left.{ }^{*}\left\{j \in Q C G_{k}\left(\Gamma^{\prime}(\lambda) ; j^{\prime}\left(\Gamma^{\prime}(\lambda)\right)\right) \mid j_{\mu(\lambda)} \notin 2 \mathbb{Z}\right\}\right) \\
& =\left(\frac{k+2}{2}\right)^{g_{1}-1} \prod_{f_{l} \in E_{\lambda}^{u}} \sin \frac{j_{l}^{\prime}+1}{2} \pi \sum_{j^{\prime}\left(E_{\lambda}^{t}\right)}(-1)^{\sum j^{\prime}\left(E_{\lambda}^{t}\right) / 2+\sum j^{\prime}\left(E_{\mu}^{u}\right) \#} Q C G_{k}\left(\Gamma^{\prime}(\lambda) \mu ; j^{\prime}\left(\Gamma^{\prime}(\lambda) \mu\right)\right) \\
& =\left(\frac{k+2}{2}\right)^{g_{1}-1} \prod_{f_{l} \in E_{\lambda}^{u}} \sin \frac{j_{l}^{\prime}+1}{2} \pi \cdot\left(\frac{k+2}{2}\right)^{g_{2}-1+m} \prod_{f_{l} \in E_{\lambda}^{u}} \sin \frac{j_{l}^{\prime}+1}{2} \pi=\gamma\left(j^{\prime}\right)\left(\frac{k+2}{2}\right)^{g-1} .
\end{aligned}
$$

Here we used the factorization property in the sixth equal sign, Proposition 6.4 in the seventh equal sign and Proposition 6.5 in the eighth equal sign.

Now we start the proof of Propositions 6.4 and 6.5.

Proof of Proposition 6.4. First note that a triple of integers $\left(k / 2, k / 2, j_{l}\right)$ satisfies the $Q C G_{k}$-condition if and only if $j_{l} \in\{0,2, \ldots, k\} \subset 2 \mathbb{Z}$, and hence one has that $Q C G_{k}^{\lambda}\left(\Gamma(\lambda) ; j^{\prime}(\Gamma(\lambda))\right) \neq \emptyset$ only if $j^{\prime}(\Gamma(\lambda)) \subset\{0,2, \ldots, k\}^{n_{1}+m} \subset 2 \mathbb{Z}^{n_{1}+m}$. Next we assume that this condition is satisfied. By the construction there are $g_{1}-1$ edges in $\operatorname{In}_{\lambda} \subset \Gamma(\lambda)$. On the other hand elements in $Q C G_{k}^{\lambda}\left(\Gamma(\lambda) ; j^{\prime}(\lambda)\right)$ have coloring $k / 2$ at edges on $\lambda$ and integer colorings $0, \ldots, k$ at edges in $\operatorname{In}_{\lambda}$. In addition such integer coloring at edges in $\operatorname{In}_{\lambda}$ can be taken arbitrary. This implies that there are $((k+2) / 2)^{g_{1}-1}$ elements in $Q C G_{k}^{\lambda}\left(\Gamma(\lambda) ; j^{\prime}(\Gamma(\lambda))\right)$. 
To show Proposition 6.5, we use the following cerebrated Verlinde formula.

Verlinde formula. Let $\Gamma$ be a unitrivalent graph and $j^{\prime}$ be a boundary coloring of $E^{u}(\Gamma)$. Then the number of $k$-admissible colorings compatible with $j^{\prime}$ is given by the following formula:

$$
d_{g, n}\left(k ; j^{\prime}(\Gamma)\right):={ }^{\#} Q C G_{k}\left(\Gamma ; j^{\prime}\right)=\left(\frac{k+2}{2}\right)^{g-1} \sum_{\nu=1}^{k+1} \frac{\prod_{f_{l} \in E^{u}(\Gamma)}\left(\sin \left[\left(j_{l}+1\right) /(k+2)\right] v \pi\right)}{(\sin [v \pi /(k+2)])^{2 g-2+n}} .
$$

Proof of Proposition 6.5. By using the factorization property and the Verlinde formula, we have the following computation:

$$
\begin{aligned}
& \sum_{j^{\prime}\left(E_{\mu}^{u}\right)}(-1)^{\sum j^{\prime}\left(E_{\mu}^{u}\right) \#} Q C G_{k}\left(\Gamma^{\prime}(\lambda)_{\mu} ; j^{\prime}\left(\Gamma^{\prime}(\lambda)_{\mu}\right)\right) \\
& =\sum_{j^{\prime}\left(E_{\mu}^{u}\right)}(-1)^{\sum j^{\prime}\left(E_{\mu}^{u}\right)}\left(\frac{k+2}{2}\right)^{g_{2}-m^{\prime}-1} \\
& \times \sum_{l=1}^{k+1} \frac{\prod_{E_{\lambda}^{t} \sqcup \overline{E_{\lambda}^{u}}}\left(\sin \left[\left(j_{v}^{\prime}+1\right) /(k+2)\right] l \pi\right) \prod_{E_{\mu}^{u}}\left(\sin ^{2}\left[\left(j_{v^{\prime}}^{\prime}+1\right) /(k+2)\right] l \pi\right)}{(\sin [l \pi /(k+2)])^{2 g_{2}-2+n}} \\
& =\left(\frac{k+2}{2}\right)^{g_{2}-m^{\prime}-1}
\end{aligned}
$$

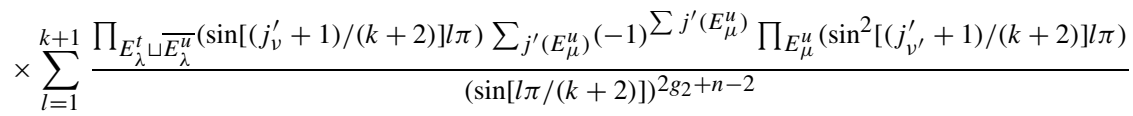

$$
\begin{aligned}
& =\left(\frac{k+2}{2}\right)^{g_{2}-1} \prod_{E_{\lambda}^{t} \sqcup E_{\lambda}^{u}} \sin \frac{j_{v}^{\prime}+1}{2} \pi .
\end{aligned}
$$

Here we used the following elementary equalities in the last equal sign:

$$
\begin{aligned}
& \sum_{j^{\prime}\left(E_{\mu}^{u}\right)}(-1)^{\sum j^{\prime}\left(E_{\mu}^{u}\right)} \prod_{E_{\mu}^{u}}\left(\sin ^{2} \frac{j_{v^{\prime}}^{\prime}+1}{k+2} l \pi\right) \\
& \quad=\sum_{j_{1}^{\prime}, \ldots, j_{m}^{\prime}}(-1)^{j_{1}^{\prime}+\cdots+j_{m}^{\prime}} \prod_{v^{\prime}=1}^{m^{\prime}}\left(\sin ^{2} \frac{j_{v^{\prime}}^{\prime}+1}{k+2} l \pi\right)= \begin{cases}{[(k+2) / 2]^{m^{\prime}},} & l=(k+2) / 2, \\
0, & l \neq(k+2) / 2 .\end{cases}
\end{aligned}
$$

Then the number in the proposition can be computed as follows:

$$
\begin{aligned}
& \left(\frac{k+2}{2}\right)^{g_{2}-1} \sum_{j^{\prime}\left(E_{\lambda}^{t}\right)}(-1)^{\sum j^{\prime}\left(E_{\lambda}^{t}\right)} \prod_{E_{\lambda}^{t} \sqcup E_{\lambda}^{u}} \sin \frac{j_{v}^{\prime}+1}{2} \pi \\
& =\left(\frac{k+2}{2}\right)^{g_{2}-1} \frac{\prod}{E_{\lambda}^{u}} \sin \frac{j_{v}^{\prime}+1}{2} \pi\left(\sum_{a=0}^{k}(-1)^{a} \sin \frac{a+1}{2} \pi\right)^{m} \\
& =\left(\frac{k+2}{2}\right)^{g_{2}-1+m} \frac{\prod}{E_{\lambda}^{u}} \sin \frac{j_{l}^{\prime}+1}{2} \pi .
\end{aligned}
$$


Remark 6.1. From the trace computation given above one can see that the external edge condition is a characterization of the Heisenberg action on $V_{2 k+4}\left(C_{\Gamma}\right)$ in the following sense. Let $\Gamma$ be a unitrivalent graph and $k$ a non-negative integer. Fix a boundary coloring $j^{\prime}$ of $\Gamma$ and let $R_{k}\left(\Gamma ; j^{\prime}\right)$ be the free $R_{k}$-module generated by the finite set $Q C G_{k}\left(\Gamma ; j^{\prime}\right)$, which is isomorphic to $V_{2 k+4}\left(C_{\Gamma} ; j^{\prime}\right)$. Take a family of maps $\alpha=\left(\alpha_{j}: H_{1}(\Gamma ; \mathbb{Z} / 2) \rightarrow R_{k}\right)$ parameterized by $j \in Q C G_{k}\left(\Gamma ; j^{\prime}\right)$. We assume that $\alpha$ satisfies the cocycle condition:

$$
\alpha_{j}\left(\lambda_{1}+\lambda_{2}\right)=\alpha_{\lambda_{2} \cdot j}\left(\lambda_{1}\right) \alpha_{j}\left(\lambda_{2}\right) \quad\left(j \in Q C G_{k}\left(\Gamma ; j^{\prime}\right), \lambda_{1}, \lambda_{2} \in H_{1}(\Gamma ; \mathbb{Z} / 2)\right) .
$$

By using such $\alpha$ we can define a homomorphism:

$$
\begin{aligned}
& \rho_{\alpha}^{(k)}: \mathcal{E}(\Gamma) \rightarrow G L\left(R_{k}\left(\Gamma ; j^{\prime}\right)\right) \\
& u^{m} \tau(\mu, \lambda) \mapsto\left(|j\rangle \mapsto A^{(k+2)^{2}(m+\mu \circ \lambda)}(-1)^{(k+1)(m+\mu \circ \lambda)+j_{\mu}} \alpha_{j}(\lambda)|\lambda \cdot j\rangle\right) .
\end{aligned}
$$

Then we have the following proposition.

Proposition 6.6. If the map $\alpha$ satisfies the cocycle condition and is compatible with the factorization of the graph, then the representation $\rho_{\alpha}^{(k)}$ on $R_{k}\left(\Gamma ; j^{\prime}\right)$ is equivalent to the representation $\rho^{(k)}$ on $V_{2 k+4}\left(C_{\Gamma} ; j^{\prime}\right)$ if and only if $\alpha=\left(\alpha_{j}\right)$ satisfies the external edge condition.

See [6] for details.

\section{Brick decomposition}

Our trace formula (Theorem 6.1) enables one to describe the dimension of the direct summands of a canonical decomposition of the TQFT-module $V_{2 k+4}(C)$ associated with the $\mathcal{E}(C)$-action. Such a decomposition is called the brick decomposition in [2].

THEOREM. [5, Theorem 1.6] Let $(C, l, c)$ be a surface with colored structure.

(i) If $k \equiv 0 \bmod 4$, then the action of $\mathcal{E}(C)$ decomposes $V_{2 k+4}(C)$ into a direct sum of subspaces $V_{2 k+4}(C, h)$, canonically associated with cohomology classes $h \in H^{1}(C-l$; $\mathbb{Z} / 2$ ).

(ii) If $k \equiv 2 \bmod 4$, then the action of $\mathcal{E}(C)$ decomposes $V_{2 k+4}(C)$ into a direct sum of subspaces $V_{2 k+4}(C, q)$, canonically associated with spin structures $q$ on $C-l$.

These subspaces are defined as follows:

$$
\begin{aligned}
& V_{2 k+4}(C, h)=\left\{v \in V_{2 k+4}(C) \mid \rho^{(k)}(\tau(a)) v=h(a) v \text { for all } a \in H_{1}(C-l ; \mathbb{Z} / 2)\right\}, \\
& V_{2 k+4}(C, q)=\left\{v \in V_{2 k+4}(C) \mid \rho^{(k)}(\tau(a)) v=q(a) v \text { for all } a \in H_{1}(C-l ; \mathbb{Z} / 2)\right\} .
\end{aligned}
$$

Here we used identifications $H^{1}(C-l ; \mathbb{Z} / 2)=\operatorname{Hom}\left(H_{1}(C-l ; \mathbb{Z} / 2), \mathbb{Z} / 2\right)$ and $\operatorname{Spin}(C-l)$ with the set of quadratic forms on $H_{1}(C-l ; \mathbb{Z} / 2)$ inducing the intersection form.

Remark 7.1. They also showed in [2] and [5] that, if $k$ is odd, then $V_{2 k+4}(C)$ is isomorphic to the tensor product of certain lower level modules as a representation of $\mathcal{E}(C)$. 
In [2] and [5] the authors gave the dimension formula for submodules $V_{2 k+4}(C, h)$ and $V_{2 k+4}(C, q)$ for a closed surface equipped with the empty link. Theorem 6.1 tells us that similar formulas hold for a surface with a non-empty link. We demonstrate the computation as follows. The computation itself is almost the same as in [2] except for the consideration of the boundary.

Now we start from a ribbon unitrivalent graph $\Gamma$. Then we have the associated closed surface $C=C_{\Gamma}$ and the isomorphism $H_{1}\left(C^{\circ} ; \mathbb{Z} / 2\right)=\Lambda_{0} / 2 \Lambda \oplus \Lambda^{*} / 2 \Lambda_{0}^{*}$, where $C^{\circ}$ is the compact surface obtained by removing open disks around the banded link in $C$.

Remark 7.2. As noted in [5], the action of $\mu=\sum \epsilon_{l} e_{l} \in \Lambda_{0}^{u} / 2 \Lambda^{u}$ represented by boundary circles is given by scalar multiplication of $(-1)^{\sum \epsilon_{l} j_{l}^{\prime}}$, and hence $V_{2 k+4}(C, h)$ (respectively $V_{2 k+4}(C, q)$ ) is zero except if $h\left(e_{l}\right)=(-1)^{j_{l}^{\prime}}$ (respectively $q\left(e_{l}\right)=(-1)^{j_{l}^{\prime}}$ ) for all boundary circles $e_{l}$. We denote by $H^{1}\left(C ; j^{\prime}\right)$ (respectively $\operatorname{Spin}\left(C ; j^{\prime}\right)$ ) the set of cohomology classes (respectively spin structures) on $C-l$ satisfying $h\left(e_{l}\right)=(-1)^{j_{l}^{\prime}}$ (respectively $q\left(e_{l}\right)=$ $\left.(-1)^{j_{l}^{\prime}}\right)$ for all $e_{l} \in \Lambda_{0}^{u} / 2 \Lambda^{u}$.

The case $k \equiv 0 \bmod 4$. We have a direct sum decomposition

$$
V_{2 k+4}(C)=V_{2 k+4}\left(C ; j^{\prime}\right)=\bigoplus_{h} V_{2 k+4}\left(C, h ; j^{\prime}\right) .
$$

The trace formula asserts that the trace takes the same value for all non-trivial non-boundary elements in $H_{1}\left(C^{\circ} ; \mathbb{Z} / 2\right)$, and is therefore invariant under the automorphism group of $H_{1}\left(C^{\circ} ; \mathbb{Z} / 2\right)$. One can check that the action of the automorphism group on $H^{1}\left(C ; j^{\prime}\right)$ is transitive. These imply that the dimension of $V_{2 k+4}(C: h)$ is the same for all nontrivial $h \in H^{1}\left(C ; j^{\prime}\right)$. We denote this dimension by $d_{g, n}^{(1)}\left(k ; j^{\prime}\right)$, and we put $d_{g, n}^{(0)}\left(k ; j^{\prime}\right):=$ $\operatorname{dim} V_{2 k+4}\left(C, 0 ; j^{\prime}\right)$. These numbers can be computed by the following two equalities:

$$
\begin{gathered}
d_{g, n}\left(k ; j^{\prime}\right)=d_{g, n}^{(0)}\left(k ; j^{\prime}\right)+\left(2^{2 g}-1\right) d_{g, n}^{(1)}\left(k ; j^{\prime}\right), \\
\gamma\left(j^{\prime}\right)\left(\frac{k+2}{2}\right)^{g-1}=\operatorname{Tr}\left(\tau_{a}\right)=d_{g, n}^{(0)}\left(k ; j^{\prime}\right)-d_{g, n}^{(1)}\left(k ; j^{\prime}\right) \quad\left(a \notin \Lambda_{0}^{u} / 2 \Lambda^{u}\right) .
\end{gathered}
$$

Note that ${ }^{\#} H^{1}\left(C ; j^{\prime}\right)=2^{2 g}$ and $d_{g, n}\left(k ; j^{\prime}\right)=\operatorname{dim} V_{2 k+4}\left(C ; j^{\prime}\right)$ is the Verlinde number. In this way for $k \equiv 0 \bmod 4$, one has that

$$
\begin{aligned}
& d_{g, n}^{(0)}\left(k ; j^{\prime}\right)=\frac{1}{2^{2 g}}\left(d_{g, n}\left(k ; j^{\prime}\right)+\left(2^{2 g}-1\right) \gamma\left(j^{\prime}\right)\left(\frac{k+2}{2}\right)^{g-1}\right), \\
& d_{g, n}^{(1)}\left(k ; j^{\prime}\right)=\frac{1}{2^{2 g}}\left(d_{g, n}\left(k ; j^{\prime}\right)-\gamma\left(j^{\prime}\right)\left(\frac{k+2}{2}\right)^{g-1}\right) .
\end{aligned}
$$

The case of $k \equiv 2 \bmod 4$. We have a direct sum decomposition

$$
V_{2 k+4}(C)=V_{2 k+4}\left(C ; j^{\prime}\right)=\bigoplus_{q} V_{2 k+4}\left(C, q ; j^{\prime}\right) .
$$

In the same way as for $k \equiv 0 \bmod 4$, the trace formula asserts that the trace is invariant under the automorphism group of $H_{1}\left(C^{\circ} ; \mathbb{Z} / 2\right)$. Fix a decomposition $H_{1}\left(C^{\circ} ; \mathbb{Z} / 2\right)=\Lambda_{0}^{u} / 2 \Lambda^{u} \oplus$ $\left(\Lambda_{0}^{u} / 2 \Lambda^{u}\right)^{\perp},\left(\Lambda_{0}^{u} / 2 \Lambda^{u}\right)^{\perp}:=H_{1}\left(C^{\circ} ; \mathbb{Z} / 2\right) /\left(\Lambda_{0}^{u} / 2 \Lambda^{u}\right)$. According to this decomposition we 
have the decomposition $q(a)=q_{1}\left(a_{1}\right) q_{2}\left(a_{2}\right)$, for $a=\left(a_{1}, a_{2}\right) \in H_{1}\left(C^{\circ} ; \mathbb{Z} / 2\right)$. Since it is enough to consider elements in $\operatorname{Spin}\left(C ; j^{\prime}\right)$, we may assume that the map $q_{1}$ is equal to the map $\sum \varepsilon_{l} e_{l} \mapsto(-1)^{\sum \varepsilon_{l} j_{l}^{\prime}}$. On the other hand there is a well-defined Arf invariant $\operatorname{Arf}\left(q_{2}\right) \in \mathbb{Z} / 2$, which parameterizes isomorphism classes of quadratic forms. In this way one knows that the dimension of $V_{2 k+4}\left(C, q ; j^{\prime}\right)$ depends only on $\operatorname{Arf}(q):=\operatorname{Arf}\left(q_{2}\right)$. For $\varepsilon=+$ or - , we put $d_{g, n}^{\varepsilon}\left(k ; j^{\prime}\right):=\operatorname{dim} V_{2 k+4}\left(C, q_{\varepsilon} ; j^{\prime}\right)$, where $q_{\varepsilon}$ has Arf invariant $\varepsilon 1 \in\{ \pm 1\}$. These numbers can be computed by the following two equalities:

$$
\begin{gathered}
d_{g, n}\left(k ; j^{\prime}\right)=2^{g-1}\left(2^{g}+1\right) d_{g, n}^{+}\left(k ; j^{\prime}\right)+2^{g-1}\left(2^{g}-1\right) d_{g, n}^{-}\left(k ; j^{\prime}\right), \\
\gamma\left(j^{\prime}\right)\left(\frac{k+2}{2}\right)^{g-1}=\operatorname{Tr}(\tau(a))=2^{g-1}\left(d_{g, n}^{+}\left(k ; j^{\prime}\right)-d_{g, n}^{-}\left(k ; j^{\prime}\right)\right) \quad\left(a \notin \Lambda_{0}^{u} / 2 \Lambda^{u}\right) .
\end{gathered}
$$

Here we used the facts that for $a \notin \Lambda_{0}^{u} / 2 \Lambda^{u}$ and $\varepsilon=+,-$, one has:

$$
{ }^{\#}\left\{q_{2}:\left(\Lambda_{0}^{u} / 2 \Lambda^{u}\right)^{\perp} \rightarrow \mathbb{Z} / 2 \mid \operatorname{Arf}\left(q_{2}\right)=0\right\}=2^{g-1}\left(2^{g}-1\right),
$$

(ii) ${ }^{\#}\left\{q_{2}:\left(\Lambda_{0}^{u} / 2 \Lambda^{u}\right)^{\perp} \rightarrow \mathbb{Z} / 2 \mid q_{2}(a)=-1, \operatorname{Arf}\left(q_{2}\right)=\varepsilon\right\}=2^{2 g-2}$.

In this way for $k \equiv 2 \bmod 4$, one has that

$$
d_{g, n}^{\varepsilon}\left(k ; j^{\prime}\right)=\frac{1}{2^{2 g}}\left(d_{g, n}\left(k ; j^{\prime}\right)+\left(\varepsilon 2^{g}-1\right) \gamma\left(j^{\prime}\right)\left(\frac{k+2}{2}\right)^{g-1}\right) .
$$

Acknowledgements. The author is grateful to G. Masbaum, who explained some details about skein theoretical computations to the author and pointed out that the description of coefficients $\delta_{j}$ could be simplified. The author would like to acknowledge CTQM, especially the director, J. E. Andersen, for their hospitality, where the author attended the lecture by G. Masbaum. The author is indebted to M. Furuta for his encouragement.

\section{REFERENCES}

[1] N. A'Campo. TQFT computations and experiments. Preprint, arXiv:math.GT/0312016.

[2] J. E. Andersen and G. Masbaum. Involutions on moduli spaces and refinements of the Verlinde formula. Math. Ann. 314 (1999), 291-326.

[3] C. Blanchet. A spin decomposition of the Verlinde formulas for type A modular categories. Comm. Math. Phys. 257(1) (2005), 1-28.

[4] C. Blanchet and G. Masbaum. Topological quantum field theories for surfaces with spin structure. Duke Math. J. 82 (1996), 229-267.

[5] C. Blanchet, N. Habegger, G. Masbaum and P. Vogel. Topological quantum field theories derived from the Kauffman bracket. Topology 34(4) (1995), 883-927.

[6] H. Fujita. External edge condition and group cohomologies associated with the quantum Clebsch-Gordan condition. Preprint, arXiv:math.GT/0708.4190.

[7] G. Masbaum and P. Vogel. 3-valent graphs and the Kauffman bracket. Pacific J. Math. 164 (1994), 361-381.

[8] T. Yoshida. An abelianization of $S U$ (2) Wess-Zumino-Witten model. Ann. of Math. (2) 164(1) (2006), 1-49.

\section{Hajime Fujita}

Department of Mathematics

Gakushuin University 1-5-1

Mejiro

Toshimaku

Tokyo 171-8588

Japan

(E-mail: hajime@math.gakushuin.ac.jp) 